\title{
Accessing long-term memory representations during visual change detection
}

\author{
Melissa R. Beck • Amanda E. van Lamsweerde
}

Published online: 10 November 2010

(C) Psychonomic Society, Inc. 2010

\begin{abstract}
In visual change detection tasks, providing a cue to the change location concurrent with the test image (postcue) can improve performance, suggesting that, without a cue, not all encoded representations are automatically accessed. Our studies examined the possibility that postcues can encourage the retrieval of representations stored in long-term memory (LTM). Participants detected changes in images composed of familiar objects. Performance was better when the cue directed attention to the post-change object. Supporting the role of LTM in the cue effect, the effect was similar regardless of whether the cue was presented during the inter-stimulus interval, concurrent with the onset of the test image, or after the onset of the test image. Furthermore, the post-cue effect and LTM performance were similarly influenced by encoding time. These findings demonstrate that monitoring the visual world for changes does not automatically engage LTM retrieval.
\end{abstract}

Keywords Visual working memory - Long term memory · Retrieval · Change detection

\section{Introduction}

It is generally agreed that between two and five attended objects, depending on the object's complexity, are stored in visual working memory (VWM) and that change detection

This research was funded by a State of Louisiana Board of Regents support fund, Research Competitiveness Subprogram Grant, to the first author.

M. R. Beck $(\square) \cdot$ A. E. van Lamsweerde

Psychology Department, Louisiana State University,

236 Audubon Hall,

Baton Rouge, LA 70808, USA

e-mail: mbeck@1su.edu failures are caused by this storage limit in VWM (Alvarez \& Cavanagh, 2004; Eng, Chen \& Jiang, 2005; Irwin, 1992; Irwin \& Andrews, 1996; Levin, Simons, Angelone \& Chabris, 2002; O'Regan, 1992; Rensink, 2002; Simons, 1996; Simons \& Levin, 1997; for review see Simons \& Rensink, 2005). However, several studies have demonstrated that change detection tasks may not accurately assess all of the information encoded into memory (Angelone, Levin \& Simons, 2003; Beck, Peterson \& Angelone, 2007; Beck \& Levin, 2003; Griffin \& Nobre, 2003; Hollingworth, 2005; Hollingworth \& Henderson, 2002; Landman, Spekreijse \& Lamme, 2003; Lepsien, Griffin, Devlin \& Nobre, 2005; Makovski, Sussman \& Jiang, 2008; Sligte, Scholte, \& Lamme, 2008; Varakin \& Levin, 2006). For example, change detection performance can be improved when a cue (an arrow pointing at the postchange object) draws attention to the post-change object (Hollingworth, 2003). Because the cue is presented after encoding, this post-cue effect is potentially aiding retrieval from long-term memory (LTM). However, several studies have not found post-cue effects (Becker, Pashler, \& Anstis, 2000; Landman et al., 2003; Luck \& Vogel, 1997; Sligte et al., 2008; Wheeler \& Treisman, 2002), suggesting that LTM representations are not automatically encoded and available for retrieval in VWM tasks. Our studies examined the conditions under which a post-cue may encourage a collaborative relationship between VWM and LTM.

In order to determine why some studies report a post-cue effect while others do not, it is important to understand the stages of processing involved in maintaining and updating visual representations (Simons, 2000; Simons \& Rensink, 2005). First, a representation of the pre-change object must be encoded and maintained in VWM and/or LTM. The post-change object must be attended, allowing for a comparison process between the pre- and post-change objects. If the representation of the pre-change object was 
encoded into LTM but is no longer available in VWM, a retrieval process must occur (Unsworth \& Engle, 2006) before comparison can occur. Finally, a decision must be made as to whether or not a change has occurred. A cue could improve change detection by improving processing in VWM (maintaining a durable representation or improved comparison), encouraging LTM retrieval, and/or reducing error in the decision process. It is important to note that these are not mutually exclusive possibilities.

\section{Cue effects on VWM}

If the cue improves processing in VWM, the cue may be orienting attention to the pre-change representation before it has faded or been overwritten by newly attended information (representation volatility hypothesis). It has been demonstrated that changes are often undetected because the pre-change representations are disrupted or lost (Beck \& Levin, 2003; Becker \& Pashler, 2002). The cue can be effective in stabilizing a representation that will otherwise fade with time or be overwritten by the test image (Averbach \& Coriell, 1961; Griffin \& Nobre, 2003; Landman et al., 2003; Lepsien et al., 2005; Makovski \& Jiang, 2007; Matsukura, Luck, \& Vecera, 2007; Sligte et al., 2008; Sperling, 1960). In these studies a cue effect was generally found when a cue was presented during the inter-stimulus interval (ISI), but not when the cue was presented with the test image, suggesting that the test image can overwrite the pre-change representation. In experiment 2 of our studies we varied the onset time of the cue to test the representation volatility hypothesis. If the representation volatility hypothesis is supported, the cue effect should be strongest when the cue occurs more closely in time to when the pre-change representation was encoded and before attention has been directed to other objects in the test image.

A post-cue may also improve processing in VWM by encouraging a more complete comparison process (comparison hypothesis). Incomplete comparison processes are more likely to result in a failure to detect small changes than large changes (Awh, Barton, \& Vogel, 2007). This suggests that detecting a small change requires a more effortful comparison than detecting a large change. If the cue acts to increase the amount of effort devoted to the comparison process, the post-cue effect should be larger for small changes. However, in Hollingworth's (2003) study, the post-cue effect was similar for both rotation changes (smaller changes) and identity changes (larger changes). Furthermore, recent research proposes that the process of comparing information in VWM to the current perceptual information occurs automatically and is preattentive, implying that an attentional cue should not affect the comparison process (Hyun, Woodman, Vogel, Hollingworth,
\& Luck, 2009). In experiment 1, size of change was quantified across several dimensions (size, average color, color variation, and orientation). If the comparison hypothesis is supported, the post-cue effect should be larger for small changes than for large changes.

\section{Cue effects on LTM}

The evidence for whether LTM representations are used to improve change detection performance has been mixed. Experts perform better than novices on change detection tasks using stimuli within their area of expertise (Reingold, Charness, Pomplun, \& Stampe, 2001; Werner \& Thies, 2000), and change detection for famous faces is better than change detection for unfamiliar faces (Jackson \& Raymond, 2008). These results are attributed to the ability to rely partially on LTM representations to improve performance. However, LTM is not necessarily used to improve change detection performance (Chen, Eng, \& Jiang, 2006). For example, performance on LTM tasks is often better than performance on change detection tasks, suggesting that LTM representations are formed, but are not necessarily retrieved during the change detection task (Angelone et al., 2003; Beck et al., 2007; Hollingworth, 2005; Hollingworth \& Henderson, 2002; Varakin \& Levin, 2006). Cues presented with the test image may help encourage LTM retrieval in situations when retrieval does not readily occur (LTM retrieval hypothesis). The goal of our experiments presented here was to demonstrate that cues can improve performance by encouraging retrieval from LTM.

Our experiments examined the LTM retrieval hypothesis by varying the number of objects encoded. If post-cues improve retrieval from LTM, then a post-cue benefit should occur primarily when the change detection task requires encoding more than four items (the approximate capacity limit of VWM). When four or fewer objects are encoded, all of the items can be stored in VWM, leading to near ceiling performance regardless of the presence of a cue. Therefore, an interaction between the number of items to be encoded and the presence of the post-cue would support the LTM retrieval hypothesis.

Our experiments also examined if the cue can encourage retrieval of LTM representations by manipulating the ability to encode LTM representations. While attending to an object generally leads to encoding a VWM representation, deeper levels of processing may be necessary for encoding a LTM representation. Familiar objects and longer encoding times are more likely to lead to the deeper levels of processing associated with strong LTM representations (Craik \& Lockhart, 1972). Furthermore, insufficient time to form detailed representations of each object is a demonstrated cause of change blindness (Brady, Konkle, 
Oliva, \& Alvarez, 2009). Hollingworth (2003) demonstrated a post-cue effect using a methodology that supports LTM representations (real world images presented for 20 seconds), while other studies used simplistic stimuli (e.g., colored boxes, digits) studied very briefly (typically less than $500 \mathrm{~ms}$ ) and did not find a post-cue effect (Becker et al., 2000; Landman et al., 2003; Luck \& Vogel, 1997; Sligte et al., 2008; Wheeler \& Treisman, 2002). In our experiments, we used complex namable objects and manipulated LTM encoding by varying the amount of time available to encode each object. If, according to the LTM retrieval hypothesis, the post-cue encourages retrieval of LTM memory representations, the post-cue effect should be stronger when the methodology supports the encoding of LTM representations. Experiment 3 demonstrates that cue effects are stronger when there is sufficient time to encode LTM representations.

\section{Cue effects on the decision process}

The post-cue could also operate by decreasing the possibility of a decision error (decision error hypothesis). Assuming that each object is represented with some noise, a change signal for a non-changing item could exceed the threshold required for reporting a change, leading to a false alarm (Wilken \& Ma, 2004). As more comparisons are made, the probability of a noisy representation for a nonchange object leading to a change response increases (Green, 1961; Hollingworth, 2003; Vogel, Woodman \& Luck, 2001; Palmer, 1990; Palmer, Ames, \& Lindsey, 1993). When a post-cue is not presented, decisions about several non-change objects in the test image may be made before a response is given and, therefore, false alarms (FAs) should increase (see Luck, 2003). However, when a postcue is presented, the probability of a decision error is reduced because only one decision is made.

\section{Experiment 1}

The goals of experiment 1 were to demonstrate a post-cue effect using a methodology supporting LTM representations and to test the comparison and decision error hypotheses. Participants completed a change detection task in which one item changed identity on half of the trials. Two blocks of trials were completed, a block in which a cue was presented with the test image and a block in which no cue appeared with the test image. Study images consisting of nameable and familiar objects were presented for $2000 \mathrm{~ms}$ with the goal of encouraging the encoding of LTM representations. Furthermore, in order to examine the post-cue effect as the amount of information encoded exceeded the capacity of VWM, study images included a circle encompassing 2, 4, 7 or 10 of the objects (pre-cue). If a change occurred, it occurred to one of the objects in the pre-cue. The results of this study supported previous research (Hollingworth, 2003) demonstrating that the postcue improves change detection performance when the methodology supports encoding of LTM representations. The comparison hypothesis was examined by comparing the post-cue effect for small and large changes and the decision error hypothesis was examined by comparing the rate of FAs on cue and no-cue trials.

Methods

\section{Participants}

Sixty students participated in this study for course credit. Half of the participants completed the post-cue block first, and half of the participants completed the no-post-cue block first. The average age of the participants was 21 years. All participants had normal or corrected-to-normal vision.

\section{Materials}

Study and test images were created using line-drawn objects with surface detail and shading (Rossion \& Pourtois, 2004; these images are a shaded and improved quality version of the Snodgrass \& Vanderwart, 1980 stimulus set and were provided courtesy of Michael J. Tarr, Brown University, Providence, RI). Each of 80 study images consisted of ten objects that were randomly chosen without replacement from a set of 240 objects and randomly placed in one of 15 locations in a $5 \times 3$ grid (see Fig. 1). Four versions of the 80 study images were created with a pre-cue, a yellowish-green circle, encompassing 2, 4, 7, or 10 of the objects.

Four test images were created for each study image: two post-cue test images and two no-post-cue test images. In the post-cue test images, a yellowish-green arrow pointed to one of the objects in the pre-cue circle (see Fig. 1). In the nopost-cue test images, the arrow was not presented. In one of two post-cue and no-post-cue images, one object was different from the object in the same location in the study image and in the other test image the object was the same. In the post-cue change image, the post-cue always pointed at the object that changed. All test images contained the pre-cue that was in the study image. The new object in the changed test images was chosen randomly from the 230 objects not already present in the study image.

Separate scripts were created so that each participant was presented with each of the 80 study images once and one of the corresponding test images, and across participants all study/test pairs were presented. In each script, half of the 80 
Fig. 1 An example of a study image with a pre-cue size of 7 and the corresponding post-cue test image. The objects were presented in grey scale and the pre-cue and post-cue were presented in a yellowish-green. In this example the lemon in the study image changes into a raccoon in the test image

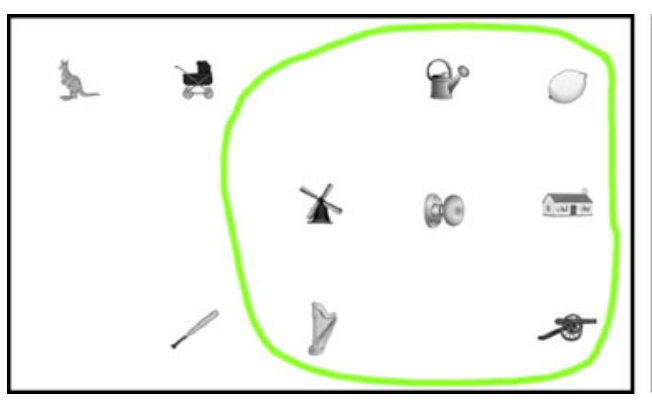

Study Image

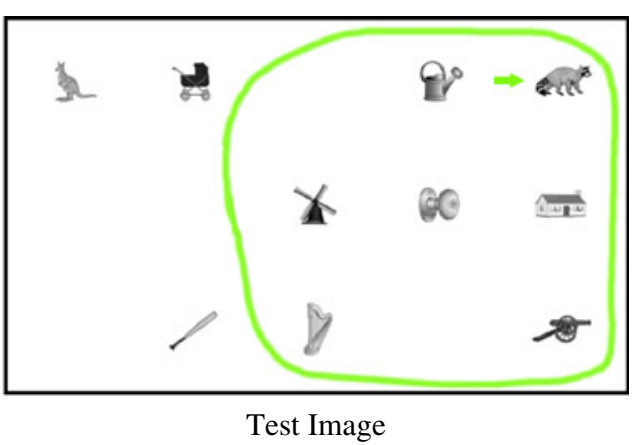

study images were paired with no-post-cue test images and the other half were paired with post-cue test images. All 40 trials with no-post-cue test images were blocked together and all 40 trials with post-cue test images were blocked together. Within each block, ten trials contained images for each of the four pre-cue sizes. Within each of these ten, five trials contained a test image with a change and five contained a test image without a change. The order of trials was random within each block.

SuperLab 4 (Cedrus Software) was used to present the stimuli and record the data. The study and test images were presented on iMac computers with 20-inch (diagonal) widescreen monitors set at a resolution of $1680 \times 1050$ and at 24-bit color depth (millions of colors). The images were $24 \times 14.5 \mathrm{~cm}$, presented at the center of a $43.5 \times 27 \mathrm{~cm}$ white screen, and subtended a $27.05^{\circ} \times 29.88^{\circ}$ visual angle from a viewing distance of approximately $47 \mathrm{~cm}$ from the screen. Objects were presented in grey scale on a white background and measured at a maximum $3.5 \mathrm{~cm} \times 2.5 \mathrm{~cm}$ $\left(4.26^{\circ} \times 3.04^{\circ}\right)$ arranged either horizontally or vertically.

The size of the change in each study/test image pair was determined across four dimensions: change in average grayscale value, change in average grayscale variation, change in size, and change in orientation. Change in grayscale value was calculated using the histogram function in Adobe PhotoShop. This histogram reports the average grayscale value for all pixels in the object. Grayscale variation in an image was calculated using the grayscale standard deviation across all pixels in the object as reported in the histogram. The size of each object was determined by the number of pixels in the object. Orientation of each object was coded as either centered, horizontal, vertical, left diagonal, or right diagonal. The difference between the preand post-change objects for each dimension was then calculated. For grayscale value, grayscale variation, and size, value was continuous and the absolute value was used. However, for orientation the value was discrete, representing either a change in orientation or no change ( 1 or 0 , respectively). All change values were converted into z-scores and then averaged for each study/test image pair. This averaged $z$-score was used to determine the size of the change in each image pair with larger z-scores representing big changes and smaller z-scores representing small changes.

\section{Procedure}

Participants completed two blocks of 40 change detection trials. For each trial, the study image was presented for $2000 \mathrm{~ms}$ and then, after a $400 \mathrm{~ms}$ ISI, the test image was presented for $2000 \mathrm{~ms}$. After the test image was presented, a screen appeared asking participants if a change had occurred. If they saw a change they were instructed to press the " 1 " key and if they did not they were instructed to press the " 2 " key.

For the no-cue block of trials, participants were instructed to determine if any of the objects in the pre-cue had changed identity. For the cue block of trials, participants were instructed that there would be an arrow in the test image pointing at one of the objects in the pre-cue and they were to determine if the object the arrow was pointed at had changed. The order of these blocks was counterbalanced across sessions. At the onset of each block the experimenter read aloud detailed instructions that were presented on the participants' computer screens.

\section{Results}

The outcomes of all analyses using proportion correct were similar to those using $A^{\prime}$. Therefore, in all experiments, proportion correct was used as the primary measure of performance.

\section{Post-cue effect}

A repeated measures analysis of variance (ANOVA) was conducted on the proportion correct with pre-cue size $(2,4$, $7,10)$ and post-cue (post-cue, no-post-cue) both as withinsubjects variables and order (post-cue first, no-post-cue first) as a between-subjects factor. There was a main effect of post-cue, $F(1,58)=21.1, M S=0.35, p<0.001$, driven by higher accuracy in the post-cue block than in the nopost-cue block. There was also a main effect of pre-cue size, $F(3,174)=150.4, M S=1.9, p<0.001$. As can be seen in Fig. 2, this effect is characterized by a tendency for 


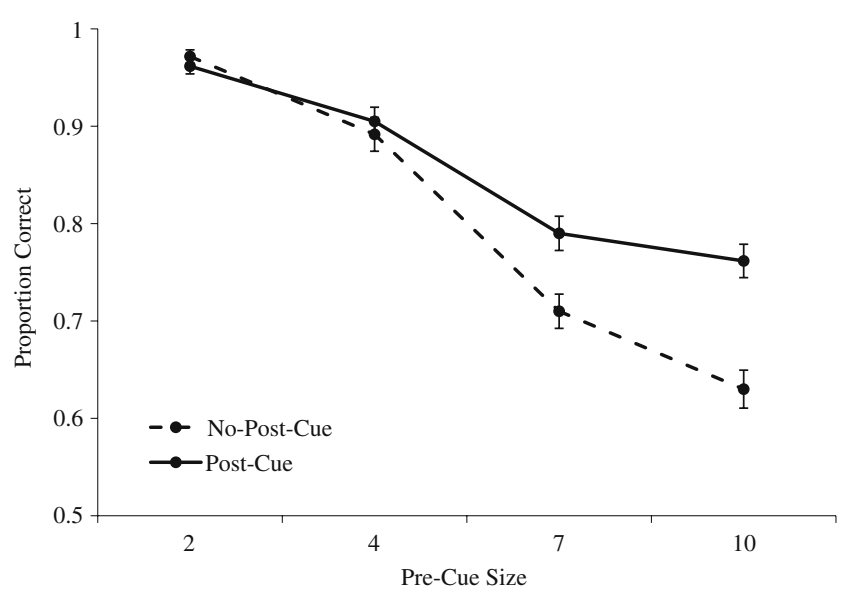

Fig. 2 Proportion correct in the post-cue (solid line) and no-post-cue (dashed line) trials for each pre-cue size in experiment 1. Bars represent standard error of the mean

performance to decrease as the size of the pre-cue increased. The post-cue/pre-cue size interaction was also significant, $F$ $(3,174)=13.2, M S=0.13, p<0.001$. This interaction was driven by no difference between the post-cue and the nopost-cue blocks for pre-cue sizes 2 and $4(p>0.28)$, but higher accuracy in the post-cue block than in the no-postcue block for pre-cue sizes $7(t(59)=-3.7, p<0.001)$ and $10(t(59)=-5.6, p<0.001)$. There was no main effect of order and no significant interactions with order. The same pattern of results was also found using $\mathrm{A}^{\prime}$ and using a capacity formula first proposed by Pashler (1988) and then modified by Cowan (2001) and Cowan, Elliott, Saults, Morey, Mattox, Hismjatullina and Conway (2005). ${ }^{1}$ The capacity estimates are presented in Table 1 and effect sizes are presented in Table 2. Hit and FA rates are presented in Appendix A.

\section{Comparison hypothesis}

Based on the average z-scores used to quantify the size of each change, the changes were rank ordered by size of change and the top $25 \%$ were categorized as large changes and the bottom $25 \%$ were categorized as small changes. The size of the post-cue effect was calculated by subtracting the proportion correct in the no-post-cue-trials from the proportion correct in the post-cue trials. The post-cue effect for small changes $(M=0.04 ; S E M=0.06)$ was not significantly larger than the post-cue effect for large changes $(M=0.07 ;$ SEM $=0.07 ; t(38)=-0.29, p=0.78)$.

\footnotetext{
${ }^{1}$ Cowan's (2001) capacity formula is $K=n(H+C R-1)$, where $K=$ the capacity estimate, $n$ equals the size of the pre-cue, $H$ is the proportion of hits on the change trials, and $C R$ is the proportion of correct rejections on the no change trials.
}

Decision-error hypothesis

A repeated measures ANOVA was conducted on the proportion of FAs with pre-cue size $(2,4,7,10)$ and postcue (post-cue, no-post-cue) both as within-subjects variables and order (post-cue first, no-post-cue first) as a between-subjects factor. There was a main effect for precue size, $F(3,174)=24.73, M S=0.47, p<0.001$, with the rate of FAs increasing as pre-cue size increased (see Appendix A). However, there was no main effect for post-cue, $F(1,58)=2.1, M S=0.04$. Therefore, the rate of FAs in the cue trials $(M=0.08 ; S E M=0.01)$ was not significantly lower than in the no-cue trials $(M=0.11 ; S E M=$ 0.01 ). There was also no significant main effect of order and no significant interactions (all $p>0.05$ ).

\section{Discussion}

A post-cue effect was found in experiment 1 , indicated by higher accuracy for the post-cue trials than for no-post-cue trials. Furthermore, change detection capacity averaged across pre-cue sizes 7 and 10 was five objects in the postcue block and three objects in the no-post-cue block. This suggests that representations of an additional two items are available for accurate change detection when a post-cue is provided. Experiment 1 did not support the representation volatility hypothesis, the comparison hypothesis, or the decision-error hypothesis. ${ }^{2}$ The post-cue effect was found even though the cue was presented concurrent with the test image. Therefore, the presentation of the test image did not overwrite the representations that were used to improve performance on the post-cue trials. This suggests that a durable representation, rather than volatile representation, was made more accessible by the cue. Furthermore, the interaction between pre-cue size and post-cue supported the LTM retrieval hypothesis but did not support the VWM hypotheses (i.e., the representational volatility and comparison hypotheses). The absence of a post-cue effect for precue sizes 2 and 4 was the result of very high no-post-cue accuracy $(M=0.93)$. This level of performance indicates that when the number of encoded items is within the capacity of VWM, cues are not needed to stabilize the representations or to encourage a more complete comparison process. The comparison hypothesis was also not supported because the post-cue did not preferentially improve change detection for small changes, and the decision-error hypothesis was not supported because the rate of FAs was similar for cue and no-cue trials.

\footnotetext{
${ }^{2}$ Blocking the post-cue and no-post-cue trials in experiment 1 may have encouraged participants to adopt different encoding strategies across the two blocks of trials. Therefore, we conducted a replication of experiment 1 with the post-cue and no-post-cue trials randomly intermixed. The results replicated those found in experiment 1 .
} 
Table 1 Capacity estimates using Cowan's (2001) formula. Average capacity estimate is shown with the standard error of the mean in parentheses. Capacities are shown for each pre-cue size $(2,4,7$, and
10) for both the no-post-cue (NPC) trials and the post-cue trials (PC) for all three experiments $(\mathrm{E} 1=$ experiment 1$)$. ISI inter-stimulus interval

\begin{tabular}{|c|c|c|c|c|c|c|c|c|}
\hline \multirow[t]{3}{*}{ Experiment } & \multicolumn{8}{|c|}{ Pre-cue sizes } \\
\hline & \multicolumn{2}{|l|}{2} & \multicolumn{2}{|l|}{4} & \multicolumn{2}{|l|}{7} & \multicolumn{2}{|l|}{10} \\
\hline & NPC & $\mathrm{PC}$ & NPC & $\mathrm{PC}$ & NPC & $\mathrm{PC}$ & NPC & $\mathrm{PC}$ \\
\hline E1 & $1.89(0.03)$ & $1.85(0.03)$ & $3.13(0.14)$ & $3.24(0.12)$ & $2.94(0.25)$ & $4.1(0.25)$ & $2.6(0.39)$ & $5.23(0.34)$ \\
\hline E2: Post-onset & $\mathrm{X}$ & $\mathrm{X}$ & $2.67(0.17)$ & $3.02(0.14)$ & $1.85(0.32)$ & $3.21(0.31)$ & $1.9(0.37)$ & $3.05(0.38)$ \\
\hline E2: ISI & $\mathrm{X}$ & $\mathrm{X}$ & $2.6(0.12)$ & $2.68(0.19)$ & $1.66(0.28)$ & $3.0(0.28)$ & $1.63(0.56)$ & $3.58(0.38)$ \\
\hline E2: Post-delayed & $\mathrm{X}$ & $\mathrm{X}$ & $2.78(0.21)$ & $2.68(0.19)$ & $.98(0.32)$ & $2.8(0.33)$ & $1.2(0.51)$ & $3(0.49)$ \\
\hline E3: $500 \mathrm{~ms}$ & $\mathrm{X}$ & $\mathrm{X}$ & $1.29(0.35)$ & $2.17(0.26)$ & $1.55(0.42)$ & $1.95(0.44)$ & $1.71(0.41)$ & $1.79(0.49)$ \\
\hline E3: $2000 \mathrm{~ms}$ & $\mathrm{X}$ & $\mathrm{X}$ & $2.57(0.21)$ & $3.11(0.23)$ & $1.85(0.42)$ & $3.15(0.39)$ & $.71(0.56)$ & $2.57(0.70)$ \\
\hline E3: $6000 \mathrm{~ms}$ & $\mathrm{X}$ & $X$ & $3.1(0.2)$ & $3.28(0.18)$ & $3.59(0.47)$ & $5.51(0.26)$ & $3.53(0.49)$ & $6.2(0.45)$ \\
\hline
\end{tabular}

\section{Experiment 2}

In Experiment 2 we further tested the possibility that the post-cue is acting on a volatile representation. A cue could improve performance by bringing attention to the postchange object before the pre-change representation has faded or before the representation has been overwritten by new information. Therefore, the volatility of the representation that benefits from a post-cue was tested by increasing the ISI to $1600 \mathrm{~ms}$ and varying the onset time of the postcue. The ISI was increased to $1600 \mathrm{~ms}$ to be sure that when the cue was provided, visual sensory memory (i.e., iconic memory) was no longer available (Averbach \& Coriell, 1961; Sperling, 1960). The cue was presented either during the ISI, concurrent with the onset of the test image, or $400 \mathrm{~ms}$ after the onset of the test image. If the post-cue effect found in Experiment 1 was caused by a volatile representation, then the size of the post-cue effect should decrease as the amount of time between presentation of the

Table 2 Cohen's d effect size for post-cue effect at pre-cue sizes 7 and 10 in all of the experiments $(\mathrm{E} 1=$ experiment 1$)$. According to Cohen (1977, 1988), all of these, except for the $500 \mathrm{~ms}$ conditions, are considered to be at least medium $(\sim 0.5)$ and mostly large $(\sim 0.8)$ effect sizes. ISI inter-stimulus interval

\begin{tabular}{lll}
\hline Experiment & Pre-cue size & \\
\cline { 2 - 3 } & 7 & 10 \\
\hline E1 & 0.59 & 0.93 \\
E2: Post-onset & 0.79 & 0.62 \\
E2: ISI & 1.02 & 0.86 \\
E2: Post-delayed & 1.3 & 0.80 \\
E3: $500 \mathrm{~ms}$ & 0.25 & 0.04 \\
E3: $2000 \mathrm{~ms}$ & 0.86 & 0.78 \\
E3: $6000 \mathrm{~ms}$ & 1.28 & 1.47 \\
\hline
\end{tabular}

study image and presentation of the cue increases (Averbach \& Coriell, 1961; Sperling, 1960). Furthermore, if attending to information in the test image easily overwrites the representations used to improve change detection when a cue is provided, presenting the cue $400 \mathrm{~ms}$ after the onset of the test image should eliminate the post-cue effect.

In experiment 1, to encourage formation of LTM representations, a nameable object changed into another namable object. It is possible that verbal encoding of these images caused the post-cue effect. Therefore, in the remaining experiments, participants repeated a string of three digits throughout the duration of the experiment.

\section{Methods}

\section{Participants}

Sixty-six participants took part in this study for course credit. Participants were randomly assigned to one of three conditions, the post-onset condition $(n=22)$, the ISI condition $(n=24)$ and the post-delayed condition $(n=20)$. The average age of the participants was 20 years. All participants had normal or corrected-to-normal vision.

\section{Materials and procedure}

The materials and procedure were the same as those used in experiment 1 except for the following changes. Only precue sizes 4, 7, and 10 were used. Participants completed 120 trials, 60 in the cue bock and 60 in the no-cue block. Within each block of trials there were 20 trials at each precue size; ten of these contained a change and ten did not.

On each trial, the study image was presented for $2000 \mathrm{~ms}$ and then, following a $1600 \mathrm{~ms}$ ISI, the test image was presented for $2000 \mathrm{~ms}$. In the post-onset condition, the post-cue was presented for the first $400 \mathrm{~ms}$ of the test image 
presentation. Then the test image was presented for the remaining $1600 \mathrm{~ms}$ without the post-cue (see Fig. 3). In the ISI condition, after $1200 \mathrm{~ms}$ of a blank screen ISI, the cue appeared for $400 \mathrm{~ms}$ on an all white background pointing at the location of the screen that would contain the change if a change occurred on the trial. In the post-delayed test condition the test image was on screen without the postcue for $400 \mathrm{~ms}$ and then the post-cue was presented with the test image for $400 \mathrm{~ms}$. The trials in the no-cue block were exactly the same across conditions.

Participants were presented with a string of three digits (randomly chosen from digits 3-9) and asked to repeat the digits throughout the entire experiment. A different set of digits was provided for each block of trials.

\section{Results}

A repeated measures ANOVA was conducted for proportion correct with pre-cue size $(4,7,10)$ and post-cue (post-cue, no-post-cue) as within subjects factors and post-cue timing (ISI, post-onset, post-delayed) and order (cue first, no-cue first) as between subjects factors. Significant main effects were found for pre-cue size, $F(2,120)=157.9, M S=1.9$, $p<0.001$, and post-cue, $F(1,60)=56.4, M S=0.42$, $p<0.001$. The interaction between pre-cue size and postcue timing was also significant, $F(1,60)=9.9, M S=0.09$,
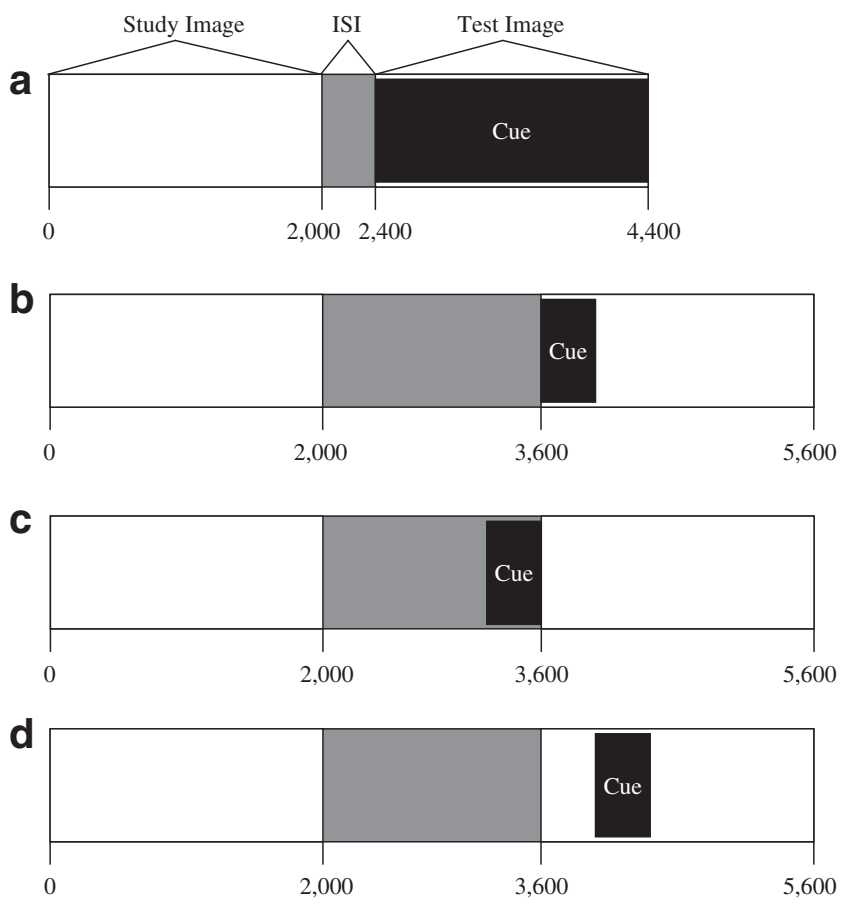

Fig. 3 Sequence of events in experiment 1 (a), and the post-onset (b), ISI (c), and post-delayed (d) conditions in experiment 2. The black box represents the presentation of the cue. In experiments 1 and 3 the post-cue was presented for the duration of the test image. In experiment 2 the cue was presented for $400 \mathrm{~ms}$. Time of presentation is presented at the bottom of each image in milliseconds $p=0.003$. The main effects for order, $F(1,60)=2.2, M S=$ $0.04, p=0.14$ and post-cue timing were not significant, $F$ $(2,60)=2.1, M S=0.03, p=0.14$. In addition, there were no significant interactions with post-cue timing (see Fig. 4).

Given the lack of a main effect for post-cue timing or any interactions with post-cue timing, performance was collapsed across levels of post-cue timing for the planned comparisons. As was found in experiment 1, there was no difference between the post-cue block and the no-post-cue block for pre-cue size $4(p=0.53)$, but there was higher change detection performance in the post-cue block for precue sizes $7(t(65)=5.8, p<0.001)$ and $10(t(65)=5.1$, $p<0.001)$.

\section{Discussion}

According to the representation volatility hypothesis, the size of the post-cue effect should decrease as the amount of time between presentation of the study image and the cue increases. However, the effect was large in all conditions and did not decrease consistently as the timing between the study image and the cue increased (see Table 2). Although the effect size (averaged across pre-cue sizes 7 and 10) decreased from the ISI condition (0.94) to the post-onset condition (0.71), contrary to the representation volatility hypothesis, the largest effect was found in post-delayed condition (1.05). These data, along with the results from experiment 1 , demonstrate that the presentation of the test image does not overwrite the representations used to improve performance when a post-cue is provided. Therefore, the post-cue effect found here is supported by a durable representation that is not easily overwritten and does not fade quickly. In addition, adding a verbal load to the change detection task did not interfere with the post-cue effect.

In experiments 1 and 2 post-cue effects were strongest when the number of encoded items exceeded VWM capacity, providing evidence in support of the LTM retrieval hypothesis, but not the volatile representation and comparison hypotheses. Experiment 3 further examined the LTM retrieval hypothesis by manipulating the amount of time participants had to encode durable representations. Previous studies reporting no improvement in performance when a post-cue is presented typically present the study scene for less than $500 \mathrm{~ms}$. Post-cue effects may have not been found in these studies because there was not sufficient time to create the complete and durable representations for which a post-cue may encourage LTM retrieval. Longer encoding time is associated with better change detection performance presumably because longer encoding times provide the opportunity to store more complete and durable representations (Brady et al., 2009). In experiment 3, we further examined the LTM retrieval hypothesis by manipulating the presentation time of the study image. If the 

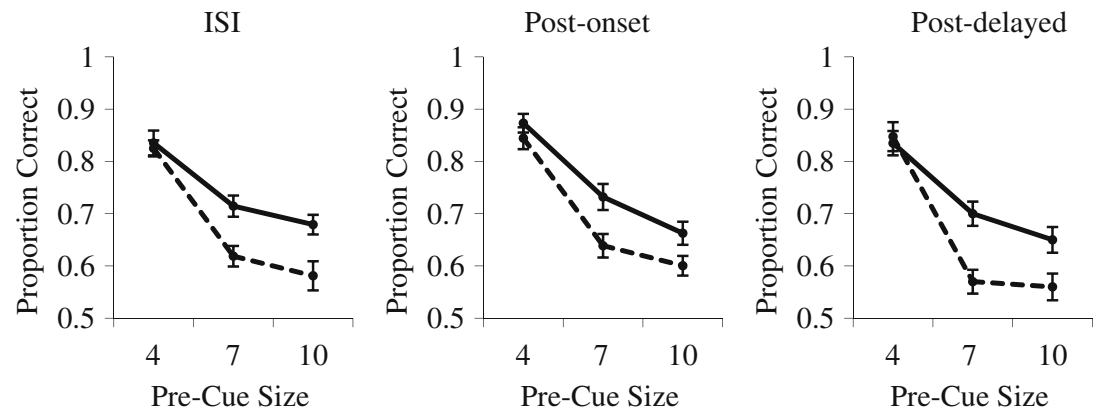

Fig. 4 Proportion correct in the post-cue (solid lines) and no-post-cue (dashed lines) trials for each pre-cue size in each condition of experiment 2. Bars represent standard error of the mean

durability of representations increases with increased encoding time and durable representations increase the post-cue effect, then a shorter presentation time should result in smaller post-cue effects.

Experiment 3 also included a LTM test for images viewed during the change detection task. If increased encoding time increases LTM representations and retrieval from LTM increases the post-cue effect, both LTM performance and the post-cue effect should improve as encoding time increases.

In order to ensure that the cue effects were not due to insufficient time to attend to all objects in the test image or to insufficient training with the change detection task, two modifications to the methodology were employed. In experiments 1 and 2 participants had only $2000 \mathrm{~ms}$ to view the test image. It is possible that this is not enough time to attend to all of the objects. If so, the cue could improve performance by bringing attention to the postchange object before the test image is removed. Therefore, in experiment 3 participants were given unlimited time to view the test image. In addition, optimal performance on a change detection task may require experience with the task and feedback. Therefore, we examined the cue effect after participants had sufficient training with the change detection task and were provided with feedback after each trial.

\section{Experiment 3}

\section{Methods}

\section{Participants}

Forty-three participants received class credit for participating and were randomly assigned to one of three conditions: the $500 \mathrm{~ms}$ condition $(n=14)$, the $2000 \mathrm{~ms}(n=14)$, and the $6000 \mathrm{~ms}$ condition $(n=15)$. The average age of the participants was 19.9 years. All participants had normal or corrected-to-normal vision.

\section{Materials and procedure}

The materials and procedure were the same as those used in experiment 2 except for the following changes. At the beginning of each block participants completed 24 training trials ( 8 trials at each set size). As in experiment 1 , the postcue was always presented with the onset of the test image and remained on screen for the duration of the test image presentation. Participants were randomly assigned to the $500 \mathrm{~ms}, 2000 \mathrm{~ms}$, or the $6000 \mathrm{~ms}$ encoding time condition. For all trials in a condition, the study images were presented for the same amount of time, either $500 \mathrm{~ms}$, $2000 \mathrm{~ms}$, or $6000 \mathrm{~ms}$, depending on the condition. The test image remained on screen until the participant responded.

Feedback was presented for $1000 \mathrm{~ms}$ following each response. If the response was correct, "Correct" appeared on the screen in green font. If the response was incorrect, "Incorrect" appeared on the screen in red font. A screen then appeared reminding participants that when the post-change scene appeared, they were to press the "p" key if they saw a change and the "q" if they did not. Participants were then instructed to press the " $\mathrm{j}$ " key to begin the next trial.

A LTM test was administered following the change detection task. In the LTM test, 120 images were presented consecutively in a random order, i.e., the 60 study images from the no-change trials in both blocks (30 from the postcue and 30 from the no-post-cue) along with 60 new images that were not viewed during the change detection task. For each image, the participant indicated if the image was old (presented during the change detection task) or new (not presented during the change detection task).

Results

\section{Change detection accuracy}

A repeated measures ANOVA was conducted for proportion correct with pre-cue size $(4,7,10)$ and post-cue (post-cue, nopost-cue) as within subjects factors and encoding time $(500 \mathrm{~ms}$, $2000 \mathrm{~ms}, 6000 \mathrm{~ms}$ ) and order (post-cue first, no-post-cue first) 
as between subjects factors. Significant main effects were found for pre-cue size, $F(2,74)=61.02, M S=0.78, p<0.001$, and post-cue, $F(1,37)=37.72, M S=0.38, p<0.001$. The main effect for encoding time was also significant, $F(2,37)=35.96$, $M S=0.72, p<0.001$. In addition, there was a significant interaction between encoding time and pre-cue size, $F(4,74)=$ $3.69, M S=0.05, p=0.009$, and a three way interaction between encoding time, pre-cue size, and post-cue, $F(4,74)=$ $2.84, M S=0.03, p=0.03$. The main effect of order was not significant, $F(1,37)=0.45, M S=0.01, p=0.51$ and there were no significant interactions with order (see Fig. 5).

Repeated measures ANOVAs were conducted on the proportion correct for each level of encoding time with precue size $(4,7,10)$ and post-cue (post-cue, no-post-cue) as within subjects variables and order (post-cue first, no-postcue first) as a between subjects factor.

In the $500 \mathrm{~ms}$ condition there was no main effect of postcue, $F(1,12)=3.25, M S=0.05, p=0.10$. There was a main effect of pre-cue size, $F(2,24)=7.63, M S=0.12, p=0.003$. No other main effects or interactions were significant (all $p>$ 0.05). Planned comparisons revealed a significant difference between the post-cue block and the no-post-cue block for precue size $4(t(13)=2.8, p=0.02)$, but no significant differences for pre-cue sizes $7(t(13)=0.69, p=0.5)$ and 10 $(t(13)=0.09, p=0.9)$.

In the $2000 \mathrm{~ms}$ condition there was a main effect of post-cue, $F(1,12)=16.31, M S=0.15, p=0.002$, and a main effect of pre-cue size, $F(2,24)=58.33, M S=0.54, p<0.001$. No other main effects or interactions were significant (all $p>0.05$ ). Planned comparisons revealed no significant difference between the post-cue block and the no-post-cue block for pre-cue size $4(t(13)=2.1, p=0.06)$, and significant differences for pre-cue sizes $7(t(13)=2.62, p=0.02)$ and $10(t(13)=2.54, p=0.03)$.

In the $6000 \mathrm{~ms}$ condition there was a main effect of postcue, $F(1,13)=32.42, M S=0.22, p<0.001$, and a main effect of pre-cue size, $F(2,26)=15.30, M S=0.18, p<0.001$. There was also a significant interaction between pre-cue size and post-cue, $F(2,26)=3.8, M S=0.03, p=0.03$. No other main effects or interactions were significant (all $p>0.05$ ).
Planned comparisons revealed no significant difference between the post-cue block and the no-post-cue block for pre-cue size $4(p>0.05)$, and significant differences for pre-cue sizes 7 $(t(14)=4.20, p<0.001)$ and $10(t(14)=4.09, p<0.001)$.

\section{LTM accuracy}

A repeated measures ANOVA with pre-cue set size $(4,7,10)$ as a within subjects factor and encoding time $(500 \mathrm{~ms}, 2000 \mathrm{~ms}$, $6000 \mathrm{~ms}$ ) as a between subjects factor was conducted on the proportion correct for the LTM trials. There was a significant main effect of pre-cue size, $F(2,80)=8.1, M S=0.05$, $p<0.001$ and a significant main effect of encoding time, $F(2$, $40)=7.9, M S=0.07, p<0.001$ (see Fig. 6). The interaction between pre-cue size and encoding time was not significant. Planned comparisons revealed that performance in the $500 \mathrm{~ms}$ condition $(M=0.57, S D=0.04)$ was significantly lower than performance in the $2000 \mathrm{~ms}$ condition $(M=0.63$, $S D=0.05), t(26)=3.36, p=0.002$. However, performance in the $6000 \mathrm{~ms}$ condition $(M=0.65, S D=0.07)$ was not significantly different from performance in the $2000 \mathrm{~ms}$ condition, $t(27)=0.94, p=0.35$. LTM performance for precue size 4 was significantly higher than performance for precue size 7 in the $500 \mathrm{~ms}$ condition, $t(13)=2.34, p=0.04$ and in the $6000 \mathrm{~ms}$ condition, $t(14)=2.5, p=0.03$. No other precue size comparisons within each level of encoding time were significant (all $p>0.05$ ).

\section{Discussion}

The post-cue effect was stronger when there was more time to encode the study items into memory and LTM performance was higher in the encoding time conditions with a post-cue effect $(2000 \mathrm{~ms}$ and $6000 \mathrm{~ms})$ than in the condition without a post-cue effect $(500 \mathrm{~ms})$. Stronger postcue effects were found when durable LTM representations could be encoded and when the number of locally attended items exceeded the capacity of VWM; this supports the hypothesis that the post-cue aids in retrieving representations from LTM.
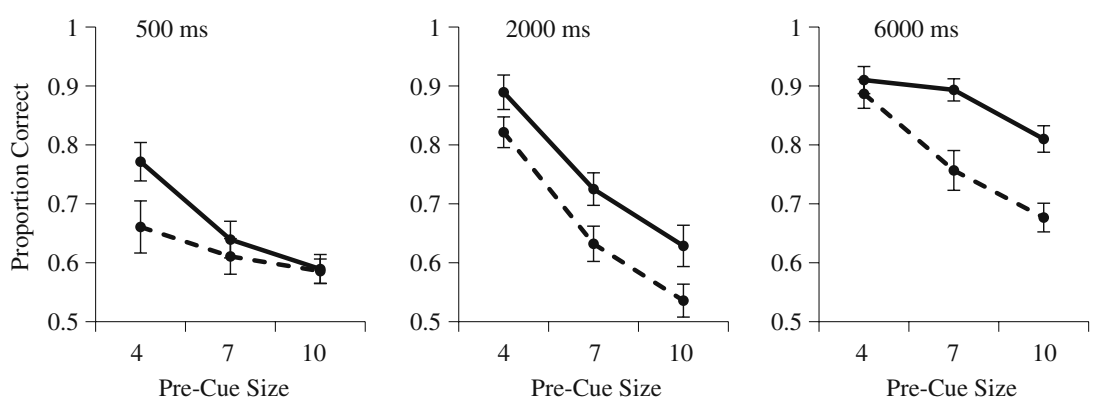

Fig. 5 Proportion correct in the post-cue (solid lines) and no-post-cue (dashed lines) trials for each pre-cue size in the $500 \mathrm{~ms}, 2000 \mathrm{~ms}$, and $6000 \mathrm{~ms}$ conditions of experiment 3. Bars represent standard error of the mean 


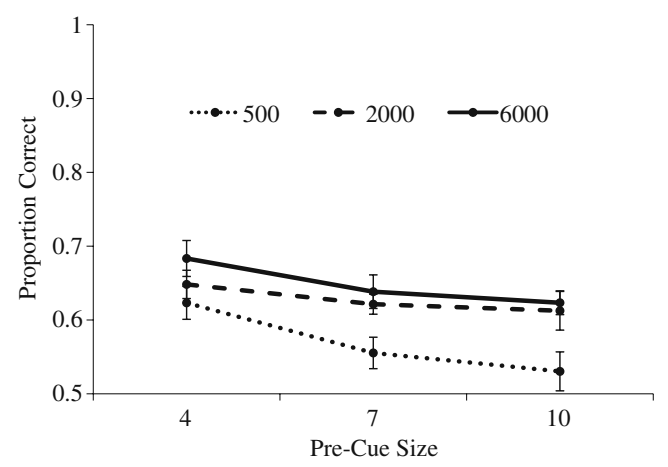

Fig. 6 Proportion correct in each encoding time condition for each pre-cue size in experiment 3 . Bars represent standard error of the mean

Interestingly, the three-way interaction between encoding time, pre-cue size, and post-cue was driven by opposite patterns for the post-cue effect across set sizes in the $500 \mathrm{~ms}$ condition compared to the $2000 \mathrm{~ms}$ and $6000 \mathrm{~ms}$ conditions. When there was only $500 \mathrm{~ms}$ to encode the items in the study image, a post-cue effect was found when there were 4 items to encode but not when there were 7 or 10 items to encode. It is unlikely that $500 \mathrm{~ms}$ is enough time to encode representations into LTM that are not also present in VWM. Therefore, this cue effect is likely due to an influence of the cue on VWM rather than LTM, supporting the possibility that the cue can operate on VWM as well as LTM.

\section{General discussion}

Across three experiments, when there was sufficient time to encode representations of the pre-change objects into LTM, a post-cue improved change detection accuracy and increased the number of items successfully monitored in a change detection task. This highly consistent pattern of results suggests that typical change detection tasks underestimate the number of objects that are encoded during presentation of the study image and that cues can be used to encourage retrieval of encoded representations from LTM. This LTM retrieval hypothesis was supported by a post-cue effect when encoding time was long enough to support LTM but not when encoding time was short. In addition, three alternative hypotheses were not supported. The representation volatility hypothesis was not supported because a post-cue effect was found even after a $1600 \mathrm{~ms}$ ISI and when the cue was presented $400 \mathrm{~ms}$ after onset of the test image. The comparison hypothesis was not supported because the post-cue benefit was not stronger for small changes than for large changes. The decision error hypothesis was not supported because FAs were not higher for no cue trials than for cue trials. Finally, the representation volatility and comparison hypotheses were not supported due to the high level of performance on the no-cue trials when the number of objects encoded into memory was within the capacity limits of VWM. Together, these studies demonstrate that, although cues may act on VWM to improve performance, post-cues can also be effective in improving change detection performance by encouraging retrieval of LTM representations.

Our studies demonstrate that change detection tasks without a post-cue may underestimate the total amount of information retained in memory from the study image. The change detection capacities found in the no-post-cue trials $(M=1.3$ averaged across pre-cue sizes 7 and 10 in the $2000 \mathrm{~ms}$ condition of experiment 3) were similar to those found in previous studies using no-post-cue change detection tasks to estimate the capacity of VWM for complex objects (1-2 objects; Alvarez \& Cavanagh, 2004; Eng et al., 2005). However, when a post-cue was provided, capacity estimates increased to 2.9 objects, suggesting that $1-2$ additional objects are stored in LTM and can be retrieved to VWM for the comparison process, given that retrieval cues are present. Post-cue effects as large as $\sim 3$ objects were found and, with increased encoding time, capacity estimates of $\sim 6$ objects were found (experiment 3, $6000 \mathrm{~ms}$ condition, precue size 10), demonstrating that in some instances more than two objects stored in LTM may be available for retrieval.

These findings are consistent with previous research demonstrating that LTM representations can be used to improve change detection performance (Beck et al., 2007; Becker \& Rasmussen, 2008; Hollingworth, 2005; Hollingworth \& Henderson, 2002; Hollingworth, Williams, \& Henderson, 2001; Ryan \& Cohen, 2004). Hollingworth (2005) demonstrated that accurate change detection was possible even after a 24-hour delay between the pre- and post-change images. Furthermore, changes can be accurately detected even when the number of items attended between attending to the pre- and post-change objects exceeds the capacity of VWM (Hollingworth, 2004; Hollingworth \& Henderson, 2002). However, Varakin and Levin (2006) demonstrated that even when both the pre- and post-change objects are attended, changes can be missed for objects that are later recognized on a LTM test. Therefore, attending to the post-change object does not guarantee that a LTM representation of that object will be retrieved and used for successful change detection. Rather, a post-cue likely encourages an effortful retrieval process that is not otherwise attempted when attending to the post-change object.

The support for the LTM retrieval hypothesis in our experiments suggests that retrieval from LTM while monitoring visual objects for changes is not necessarily an automatic process. In order for information in LTM to be available to VWM, the information in LTM has to reach a particular level of activation (Anderson, 1983; Cowan, 1988). Our results suggest that this level of activation is met more readily when a post-cue is provided. Alternatively, the lack of support for the representation volatility and comparison hypotheses in our experiments suggests that for the information in VWM, 
these processes can occur in the absence of a cue. Therefore, the ability to attend, compare and maintain representations in VWM may be more automatic than the ability to retrieve LTM representations.

The decision noise hypothesis was not supported in our experiments. Across all three experiments, in all but one instance (experiment 2, post-delayed condition, pre-cue size 7), FAs were not significantly higher for the no-post-cue trials than for the post-cue trials (see Appendix A). In the postdelayed condition of experiment 2, FAs should be most likely to occur in the post-cue trials due to an increase in decision errors. The post-cue was delayed until $400 \mathrm{~ms}$ after the onset of the test image, allowing decisions about non-cued objects to be made before the cue was presented, leading to higher levels of decision errors. However, this increase in decision errors did not decrease the post-cue effect (see Table 2). In addition, in the $500 \mathrm{~ms}$ condition of experiment 3 , a post-cue main effect was not found even though the same number of decisions needed to be made as in the other conditions. Finally, if a decrease in decision errors was the cause of the post-cue effect, then previous studies using simplistic stimuli should have also found a post-cue effect. Although a similar number of decisions had to be made as in our studies ( 8 objects in Becker et al., 2000 and Landman et al., 2003 and 12 objects in Luck \& Vogel, 1997), none of these previous studies found a post-cue effect. For these reasons, it is unlikely that our findings can be explained by a decrease in decision errors in the post-cue trials.

There are a few other possible explanations that need to be considered. First, it is possible that rather than retrieving items from LTM, not all items in VWM are being compared. Postcue effects demonstrate a larger capacity durable representation available to VWM but not active in VWM. Therefore, representations previously actively available in VWM may become less available, not because they were overwritten by newly attended information (Beck \& Levin, 2003; Lakha \& Wright, 2004; Simons, 2000), but because they are outside the focus of attention (Cowan, 1988, 1999, 2001). The cue could serve to bring the representation into the focus of attention within VWM rather than serving to transfer the representation from LTM to VWM. However if, as recent research suggests, the process of comparing information in VWM to the current perceptual information occurs automatically and is preattentive, then the attentional cue should not affect the change detection process for items within VWM (Hyun, Woodman, Vogel, Hollingworth, \& Luck, 2009).

Further lack of support for the possibility of the post-cue effect resulting from comparison of objects in VWM that are not otherwise compared comes from the studies that failed to find a post-cue effect using more simplistic stimuli and short encoding times (Becker et al., 2000; Landman et al., 2003; Luck \& Vogel, 1997; Wheeler \& Treisman, 2002). It does not seem likely that comparison of all items stored in VWM could occur for representations of more simplistic stimuli but not for representations of more complex stimuli. If this is the case, it would suggest that a post-cue increases VWM capacity estimates for complex objects but not simplistic objects, suggesting that previous studies showing smaller capacity estimates for complex objects in VWM using a change detection task (Alvarez \& Cavanagh, 2004; Eng et al., 2005) may have actually been measuring the extent to which the comparison process is exhaustive for simplistic versus complex objects. Furthermore, in experiment 3, a post-cue effect was not found for the $500 \mathrm{~ms}$ presentation of the study image. It seems unlikely that representations of complex objects in VWM would be compared for short encoding times, but not for long encoding times. Therefore, although the possibility of the post-cue improving access or comparison of objects stored in VWM may not be entirely ruled out, the LTM retrieval hypothesis remains the most probable explanation for the results.

Our findings should be contrasted with the studies reporting that a cue only improves the representation of simplistic stimuli when attention is drawn to the representation during the ISI (retro-cue effect: Becker et al., 2000; Landman et al., 2003; Makovski \& Jiang, 2007; Makovski et al., 2008). We found that cues presented during the ISI and cues presented concurrent with the test image improve change detection performance. The cue effect found in the ISI condition of experiment 2 may be due to direction of attention to a volatile representation or it may be caused by retrieval from LTM. There was a small tendency for effect sizes to be larger in the ISI condition of experiment 2, suggesting that the mechanism underlying the cue effect may be different from the mechanism underlying the post-cue effect. In addition, performance on the cue trials was higher than performance on the no-cue trials in the pre-cue size 4, $500 \mathrm{~ms}$ condition of experiment 3 . This also suggests that the cue may have acted on more volatile representations. However, the goal of our experiments was not to rule out the possibility that retro-cue effects occur; several studies have clearly shown that they do occur. Rather, the goal was to demonstrate the conditions under which a cue effect that results from the retrieval of durable LTM representations can occur.

In conclusion, traditional change detection tasks have been used extensively to estimate the capacity of VWM. While this may be an accurate measure of what is currently held in VWM, it is not an accurate measure of all the information retained from viewing a visual scene. Information that was previously stored in VWM can be made available for the comparison process given adequate retrieval cues are provided. In the absence of a retrieval cue, this information may not be used in tracking visual information over time. Our studies add to our understanding of the relationship between VWM and LTM and how they interact toward the goal of perceiving the visual world over time. 


\section{Appendix A}

Table 3 Hit and false alarm (FA) rates for each pre-cue size $(2,4,7$, and 10$)$ for both the nopost-cue (NPC) trials and the post-cue trials (PC) for all three experiments $(\mathrm{E} 1=$ experiment 1). It is important to note that, when separated by type of change AND set size, the hit and FA rates for the large and small changes in experiment 1 data are difficult to interpret because the number of trials included in each cell is small. For example, only four trials are included for the FA mean of the small changes/post-cue/pre-cue size 4

ISI inter-stimulus interval, $F A$ false alarm

\begin{tabular}{|c|c|c|c|c|c|c|c|c|c|}
\hline \multirow[t]{3}{*}{ Experiment } & \multirow[t]{3}{*}{ Rates } & \multicolumn{8}{|c|}{ Pre-cue size } \\
\hline & & \multicolumn{2}{|l|}{2} & \multicolumn{2}{|l|}{4} & \multicolumn{2}{|l|}{7} & \multicolumn{2}{|l|}{10} \\
\hline & & NPC & $\mathrm{PC}$ & NPC & $\mathrm{PC}$ & NPC & $\mathrm{PC}$ & NPC & $\mathrm{PC}$ \\
\hline \multirow[t]{2}{*}{ E1: All trails } & Hit & 0.97 & 0.95 & 0.84 & 0.86 & 0.42 & 0.65 & 0.51 & 0.68 \\
\hline & FA & 0.03 & 0.03 & 0.06 & 0.05 & 0.18 & 0.13 & 0.16 & 0.13 \\
\hline \multirow[t]{2}{*}{ E1: Small changes } & Hit & 0.97 & 0.97 & 0.82 & 0.85 & 0.69 & 0.63 & 0.31 & 0.79 \\
\hline & FA & 0.07 & 0.08 & 0.09 & 0.03 & 0.10 & 0.14 & 0.12 & 0.06 \\
\hline \multirow[t]{2}{*}{ E1: Large changes } & Hit & 0.95 & 0.96 & 0.92 & 0.89 & 0.57 & 0.83 & 0.47 & 0.57 \\
\hline & FA & 0.03 & 0.06 & 0.07 & 0.06 & 0.23 & 0.14 & 0.06 & 0.05 \\
\hline \multirow[t]{2}{*}{ E2: Post-onset } & Hit & $\mathrm{X}$ & $\mathrm{X}$ & 0.77 & 0.86 & 0.49 & 0.69 & 0.51 & 0.56 \\
\hline & FA & & & 0.11 & 0.11 & 0.23 & 0.23 & 0.32 & 0.26 \\
\hline \multirow[t]{2}{*}{ E2: ISI } & Hit & $\mathrm{X}$ & $X$ & 0.75 & 0.76 & 0.47 & 0.65 & 0.48 & 0.58 \\
\hline & FA & & & 0.10 & 0.09 & 0.23 & 0.22 & 0.32 & 0.23 \\
\hline \multirow[t]{2}{*}{ E2: Post-delayed } & Hit & $\mathrm{X}$ & $\mathrm{X}$ & 0.75 & 0.78 & 0.43 & 0.57 & 0.42 & 0.56 \\
\hline & FA & & & 0.06 & 0.11 & 0.29 & 0.17 & 0.30 & 0.26 \\
\hline \multirow[t]{2}{*}{$\mathrm{E} 3: 500 \mathrm{~ms}$} & Hit & $X$ & $\mathrm{X}$ & 0.48 & 0.74 & 0.42 & 0.54 & 0.42 & 0.56 \\
\hline & FA & & & 0.16 & 0.2 & 0.2 & 0.26 & 0.25 & 0.38 \\
\hline \multirow[t]{2}{*}{ E3: $2000 \mathrm{~ms}$} & Hit & $X$ & $X$ & 0.72 & 0.90 & 0.48 & 0.69 & 0.40 & 0.57 \\
\hline & FA & & & 0.08 & 0.12 & 0.21 & 0.24 & 0.33 & 0.33 \\
\hline \multirow[t]{2}{*}{ E3: $6000 \mathrm{~ms}$} & Hit & $\mathrm{X}$ & $\mathrm{X}$ & 0.83 & 0.91 & 0.66 & 0.90 & 0.58 & 0.81 \\
\hline & FA & & & 0.06 & 0.09 & 0.15 & 0.11 & 0.23 & 0.19 \\
\hline
\end{tabular}

\section{References}

Alvarez, G., \& Cavanagh, P. (2004). The capacity of visual short term memory is set both by visual information load and by number of objects. Psychological Science, 15(2), 106-111. doi:10.1111/ j.0963-7214.2004.01502006.x.

Anderson, J. R. (1983). The architecture of cognition. Cambridge: Harvard University Press.

Angelone, B. L., Levin, D. T., \& Simons, D. J. (2003). The relationship between change detection and recognition of centrally attended objects in motion pictures. Perception, 32, 947-962. doi: 10.1068/p5079.

Averbach, E., \& Coriell, A. S. (1961). Short-term memory in vision. Bell System Technical Journal, 40, 309-328.

Awh, E., Barton, B., \& Vogel, E. (2007). Visual working memory represents a fixed number of items, regardless of complexity. Psychological Science, 18, 622-628. doi:10.1111/j.14679280.2007.01949.x.

Beck, M. R., \& Levin, D. T. (2003). The role of representational volatility in recognizing pre- and post-change objects. Perception \& Psychophysics, 65(3), 458-468.

Beck, M. R., Peterson, M. S., \& Angelone, B. L. (2007). The roles of encoding, retrieval, and awareness in change detection. Memory \& Cognition, 16, 31-51.

Becker, M. W., \& Pashler, H. (2002). Volatile visual representations: Failing to detect changes in recently processed information. Psychonomic Bulletin \& Review, 9(4), 744-750.
Becker, M. W., Pashler, H., \& Anstis, S. M. (2000). The role of iconic memory in change-detection tasks. Perception, 29, 273-286.

Becker, M. W., \& Rasmussen, I. P. (2008). Guidance of attention to objects and locations by long-term memory of natural scenes. Journal of Experimental Psychology. Learning, Memory, and Cognition, 34(6), 1325-1338. doi:10.1037/a0013650.

Brady, T. F., Konkle, T., Oliva, A., \& Alvarez, G. A. (2009). Detecting changes in real-world objects: The relationship between long-term memory and change detection. Communicative \& Integrative Biology, 2(1), 1-3.

Chen, D., Eng, H. Y., \& Jiang, Y. (2006). Visual working memory for trained and novel polygons. Visual Cognition, 14(1), 37-54. doi:10.1080/13506280544000282.

Cohen, J. (1977). Statistical power analysis for the behavioral sciences (revised edition). New York: Academic Press.

Cohen, J. (1988). Statistical power analysis for the behavioral sciences (2nd ed.). Hillsdale: Erlbaum.

Cowan, N. (1988). Evolving conceptions of memory storage, selective attention, and their mutual constraints within the human information processing system. Psychological Bulletin, 104, 163-191. doi:10.1037/0033-2909.104.2.163.

Cowan, N. (1999). An embedded-processes model of working memory. In A. Miyake \& P. Shah (Eds.), Models of working memory: Mechanisms of active maintenance and executive control (pp. 62-101). Cambridge: Cambridge University Press.

Cowan, N. (2001). The magical number 4 in short-term memory: A reconsideration of mental storage capacity [Target article and 
commentaries]. The Behavioral and Brain Sciences, 24, 87-185. doi:10.1017/S0140525X01003922.

Cowan, N., Elliott, E. M., Saults, J. S., Morey, C. C., Mattox, S. M., Hismjatullina, A., et al. (2005). On the capacity of attention: Its estimation and its role in working memory and cognitive aptitudes. Cognitive Psychology, 51, 42-100. doi:10.1016/j. cogpsych.2004.12.001.

Craik, F. I. M., \& Lockhart, R. S. (1972). Levels of processing: A framework for memory research. Journal of Verbal Learning and Verbal Behaviour, 11, 671-684. doi:10.1016/S0022-5371(72)80001-X.

Eng, H. Y., Chen, D., \& Jiang, Y. (2005). Visual working memory for simple and complex visual stimuli. Psychonomic Bulletin \& Review, 12(6), 1127-1133.

Green, D. M. (1961). Detection of auditory sinusoids of uncertain frequency. The Journal of the Acoustical Society of America, 33, 897-903. doi:10.1121/1.1908839.

Griffin, I. C., \& Nobre, A. C. (2003). Orienting attention to location in internal representations. Journal of Cognitive Neuroscience, 15 (8), 1176-1194. doi:10.1162/089892903322598139.

Hollingworth, A. (2003). Failures of retrieval and comparison constrain change detection in natural scenes. Journal of Experimental Psychology: Human Perception and Performance, 29(2), 388-403. doi:10.1037/0096-1523.29.2.388.

Hollingworth, A. (2004). Constructing visual representations of natural scenes: The roles of short- and long-term visual memory. Journal of Experimental Psychology: Human Perception and Performance, 30(3), 519-537. doi:10.1037/0096-1523.30.3.519.

Hollingworth, A. (2005). The relationship between online representations of a scene and long-term scene memory. Journal of Experimental Psychology. Learning, Memory, and Cognition, 31(3), 396-411. doi:10.1037/0278-7393.31.3.396.

Hollingworth, A., \& Henderson, J. M. (2002). Accurate visual memory for previously attended objects in natural scenes. Journal of Experimental Psychology: Human Perception and Performance, 28(1), 113-136. doi:10.1037/0096-1523.28.1.113.

Hollingworth, A., Williams, C. C., \& Henderson, J. M. (2001). To see and remember: Visually specific information is retained in memory from previously attended objects in natural scenes. Psychonomic Bulletin \& Review, 8(4), 761-768.

Hyun, J., Woodman, G. F., Vogel, E. K., Hollingworth, A., \& Luck, S. J. (2009). The comparison of visual working memory representations with perceptual inputs. Journal of Experimental Psychology: Human Perception and Performance, 35(4), 1140-11160. doi: $10.1037 / \mathrm{a} 0015019$.

Irwin, D. E. (1992). Memory for position and identity across eye movements. Journal of Experimental Psychology. Learning, Memory, and Cognition, 18(2), 307-317. doi:10.1037/0278-7393.18.2.307.

Irwin, D. E., \& Andrews, R. V. (1996). Integration and accumulation of information across saccadic eye movements. In T. Inui \& J. L. McCleland (Eds.), Attention and performance XVI: Information integration in perception and communication. Cambridge, MA: MIT Press.

Jackson, M. C., \& Raymond, J. E. (2008). Familiarity enhances visual working memory for faces. Journal of Experimental Psychology: Human Perception and Performance, 34(3), 556-568. doi:10.1037/0096-1523.34.3.556.

Lakha, L., \& Wright, M. J. (2004). Capacity limitations of visual memory in two-interval comparison of garbor arrays. Vision Research, 44, 1707-1716. doi:10.1016/j.visres.2004.02.006.

Landman, R., Spekreijse, H., \& Lamme, V. A. F. (2003). Large capacity storage of integrated objects before change blindness. Vision Research, 43, 149-164. doi:10.1016/S0042-6989(02)00402-9.

Lepsien, J., Griffin, I. C., Devlin, J. T., \& Nobre, A. C. (2005). Directing attention in mental representations: Interactions between attentional orienting and working-memory load. Neuroimage, 26, 733-743.
Levin, D. T., Simons, D. J., Angelone, B. L., \& Chabris, C. F. (2002). Memory for centrally attended changing objects in an incidental real-world change detection paradigm. British Journal of Psychology, 93(3), 289-302. doi:10.1348/000712602760146224.

Luck, S. J. (2003). Visual short-term memory. In S. J. Luck \& A. Hollingworth (Eds.), Visual Memory. New York: Oxford Press.

Luck, S. J., \& Vogel, E. K. (1997). The capacity of visual working memory for features and conjunctions. Nature, 390, 279-281. doi: $10.1038 / 36846$.

Makovski, T., \& Jiang, Y. (2007). Distributing versus focusing attention in visual short-term memory. Psychonomic Bulletin \& Review, 14(6), 1072-1078.

Makovski, T., Sussman, R., \& Jiang, Y. (2008). Orienting attention in visual working memory reduces interference from memory probes. Journal of Experimental Psychology. Learning, Memory, and Cognition, 34(2), 369-380. doi:10.1037/0278-7393.34.2.369.

Matsukura, M., Luck, S. J., \& Vecera, S. P. (2007). Attention effects during visual short-term memory maintenance: Protection or prioritization. Perception \& Psychophysics, 69(8), 1422-1434.

O'Regan, J. K. (1992). Solving the 'real' mysteries of visual perception: The world as an outside memory. Canadian Journal of Psychology, 46(3), 461-488. doi:10.1037/h0084327.

Palmer, J. (1990). Attentional limits on the perception and memory of visual information. Journal of Experimental Psychology: Human Perception and Performance, 16(2), 3332-3350. doi:10.1037/ 0096-1523.16.2.332.

Palmer, J., Ames, C. T., \& Lindsey, D. T. (1993). Measuring the effect of attention on simple visual search. Journal of Experimental Psychology: Human Perception and Performance, 19(1), 108130. doi:10.1037/0096-1523.19.1.108.

Pashler, H. (1988). Familiarity and visual change detection. Perception \& Psychophysics, 44, 369-378.

Rensink, R. A. (2002). Change detection. Annual Review of Psychology, 53, 245-277. doi:10.1146/annurev.psych.53.100901.135125.

Reingold, E. M., Charness, N., Pomplun, M., \& Stampe, D. M. (2001). Visual span in expert chess players: Evidence from eye movements. Psychological Science, 12, 48-55. doi:10.1111/1467-9280.00309.

Rossion, B., \& Pourtois, G. (2004). Revisiting Snodgrass and Vanderwart's object pictorial set: The role of surface detail in basic-level object recognition. Perception, 33, 217-236. doi:10.1068/p5117.

Ryan, J. D., \& Cohen, N. J. (2004). The nature of change detection and online representations of scenes. Journal of Experimental Psychology: Human Perception and Performance, 30(5), 9881015. doi:10.1037/0096-1523.30.5.988.

Simons, D. J. (1996). In sight, out of mind: When object representations fail. Psychological Science, 7, 301-305. doi:10.1111/ j.1467-9280.1996.tb00378.x.

Simons, D. J. (2000). Current approaches to change blindness. Visual Cognition, 7(1-3), 1-15. doi:10.1080/135062800394658.

Simons, D. J., \& Levin, D. T. (1997). Change blindness. Trends in Cognitive Sciences, 1(7), 261-267. doi:10.1016/S1364-6613(97) 01080-2.

Simons, D. J., \& Rensink, R. A. (2005). Change blindness: Past, present, and future. Trends in Cognitive Sciences, 9(1), 16-20. doi:10.1016/j.tics.2004.11.006.

Sligte, I. G., Scholte, S., \& Lamme, V. A. F. (2008). Are there multiple visual short-term memory stores? PLOS ONE, 3(2), e1699.

Snodgrass, J. G., \& Vanderwart, M. (1980). A standardized set of 260 pictures: Norms for name agreement, image agreement, familiarity, and visual complexity. Journal of Experimental Psychology: Human Learning and Memory, 6(2), 174-215. doi:10.1037/02787393.6.2.174.

Sperling, G. (1960). The information available in brief visual presentations. Psychological Monographs, 74, 1-29.

Unsworth, N., \& Engle, R. W. (2006). Simple and complex memory spans and their relation to fluid abilities: Evidence from list- 
length effects. Journal of Memory and Language, 54, 68-80. doi:10.1016/j.jml.2005.06.003.

Varakin, D., \& Levin, D. T. (2006). Change blindness and visual memory: Visual representations get rich and act poor. British Journal of Psychology, 97(1), 51-77. doi:10.1348/000712605X68906.

Vogel, E. K., Woodman, G. F., \& Luck, S. J. (2001). Storage of features, conjunctions, and objects in visual working memory. Journal of Experimental Psychology: Human Perception and Performance, 27(1), 92-114. doi:10.1037/0096-1523.27.1.92.
Werner, S., \& Thies, B. (2000). Is "change blindness" attenuated by domain-specific expertise? An expert-novices comparison of change detection in football images. Visual Cognition, 7, 163173. doi: $10.1080 / 135062800394748$.

Wheeler, M. E., \& Treisman, A. (2002). Binding in short-term visual memory. Journal of Experimental Psychology: General, 131(1), 48-64. doi:10.1037/0096-3445.131.1.48.

Wilken, P., \& Ma, W. J. (2004). A detection theory account of change detection. Journal of Vision, 4, 1120-1135. doi:10.1167/4.12.11. 